\title{
Design of Tangible Games for Children Undergoing Occupational and Physical Therapy
}

\author{
Robby van Delden ${ }^{1}$, Pauline Aarts ${ }^{2}$, and Betsy van Dijk ${ }^{1}$ \\ 1 Human Media Interaction, University of Twente, Enschede, The Netherlands \\ P.O. Box 217, 7500 AE, Enschede, The Netherlands \\ $\{r$.w.vandelden, e.m.a.g.vandijk\}@utwente.nl \\ 2 Department of Paediatric Rehabilitation, Sint Maartenskliniek, \\ Hengstdal 3, 6574 NA Ubbergen (near Nijmegen), The Netherlands \\ p.aarts@maartenskliniek.nl
}

\begin{abstract}
Games can offer an entertaining alternative to repetitive tasks. In this paper, we propose the use of tangible interactive games for the repetitive training of upper limbs in the therapy of children with Cerebral Palsy (CP). We obtained promising results. The total of four created games succeeded in triggering all the to-be-trained movements properly and in a motivating and entertaining way. A physical quiz game was especially successful as children kept on playing the game making the proper movements without additional encouragement or instructions of the therapists or researchers. These results indicate that in this kind of occupational or physical therapy, there is additional value in using tangible interactive games. Furthermore, the research shows the importance of including the therapists in the design of games and we report on several ways to achieve that.
\end{abstract}

Keywords: Participatory design, games for therapy, tangible games, special user groups, children, cerebral palsy.

\section{Introduction}

Hitting a button for almost a thousand times does not sound like much fun. However, over the last three decades children have had fun doing this in the form of computer games. Most forms of physical therapy for children are also repetitive in nature, especially for children with unilateral Cerebral Palsy $(\mathrm{CP})$. Unilateral $\mathrm{CP}$ is a disorder of posture and/or motor function due to brain damage in the first year of life. In the unilateral type of $\mathrm{CP}$, the right or left side of the body is affected. Therapy for $\mathrm{CP}$ children can be effective but can also be boring at times because of this repetitive nature [1]. Using interactive games could reasonably be expected to help in bringing additional motivation and so more enjoyable therapy.

The TagTiles platform can be used for creating such interactive games with tangibles; it is a system that can automatically localise multiple objects on its 
surface by using RFID tags. This system gives feedback with full colour LEDs and with playing sounds. In previous research, TagTiles games were created for therapy for CP patients [15]. However, these games were not implemented as the proper movements were not triggered enough and compensating movements were observed too often. Nonetheless, the use of entertaining interactive tangible games built for the TagTiles system was promising according to the therapists. That new games for the TagTiles system were not developed earlier by therapists themselves is probably due to a lack of time, effort and know-how from therapists to develop games that are suitable 13 .

Children with unilateral CP are inclined to make compensating movements. In other words, they prevent certain movements that are hard for them and thereby further diminish their capability of performing these movements. Therapy especially focuses on training the fine motor skills of the upper body. Therapists have a good insight in how to trigger the correct movements and what the preferences are of the children. We thus chose to use a participatory design approach. At the same time, we had to keep in mind that there is limited time available for non-therapy activities by the therapists and children 13 .

These children may also suffer from forms of mental retardation and low selfesteem. Although several guidelines for developing motivating games exist (e.g. 16]), they do not explicitly take into account the special needs of these children. Thus, during this research we looked into the use of sounds and evaluated and compared different versions of tangible games for therapy along the dimensions of challenge and interpersonal competition.

In related work, several attempts of using interactive games in therapy are reported and results seem promising [2 5 7 9]. However, to our knowledge there are no successful and well-tested implementations of interactive tangible games for physical therapy of CP patients focusing on the upper limbs. This was a main motivator for our research.

Over one and a half years of research, several design tools and techniques were used to deal effectively with this challenge. We used a participatory design approach, incorporating the therapists knowledge and the children's interests. This was done in two main rounds of development leading to four basic games. The games were evaluated in a mainly qualitative way with a total of seven therapists and eighteen different children undergoing therapy.

The remainder of this paper is structured as follows. In the following section, we summarise related work on motivating games and games for therapy. Section 3 describes the methodology and test setup. Section 4 describes the games developed. This is followed in Section 5 with the results. The paper concludes with a discussion and proposals for future work.

\section{Motivation and Games for Physical Therapy}

Adding motivation and turning cumbersome activities into fun and engaging tasks for children is challenging. Malone and Lepper pioneered research in this field [16]. They worked on fun and motivation in educational computer games. 
We will only mention some of their findings as their work has already been analysed, rewritten and complemented several times (e.g. [12 17]).

Their analysis resulted in four individual criteria: Challenge, Curiosity, Fantasy and Control. Three interpersonal criteria were also stated: Cooperation, Competition and Recognition (meaning appreciation). These aspects still seem to cover the most important aspects of what makes games fun and can be found in one way or another in most of the recently developed frameworks (e.g. 14 ).

TagTiles has been targeted as a platform for therapy before by $\mathrm{Li}$ et al. 15. They developed three games for TagTiles. Results were promising but they overestimated the physical abilities of the children and the games lacked the capability to withstand possible compensations by the children to circumvent the to-be-trained movements. This mainly involves circumventing difficult movements. For example, using their non-affected arm to grab something or bending their affected wrist downward (palmar) to an extreme extent. However, "All participants said they enjoyed playing the game" and "Therapists were happy to observe children engage in the desired movements spontaneously, something that they said was not common in their standard therapy sessions." [15, p190].

Getting games to be used in therapy also requires incorporating the context and therapist's view. Applicable requirements and effective methodology for this context can be found in [2]. Based on observation of therapy and a small-scale implementation, the following was emphasised: the importance of limited preparation and start up time, adaptability and adaptivity, simplicity and (where possible) automated logging.

Several studies underline that using interactive video games for therapy and rehabilitation can be useful [9]. For instance, playing the basic games on the Wii had a positive result in motor function of the legs, such as increased gait distance and increased postural control of a 13-year old CP patient suffering from double leg impairment 7 . Another small-scale study of four children using a camera-based input virtual reality application for two weeks, showed it is also possible to improve "reaching kinematics" of children with CP by using games [5]. The therapy of our target group mainly focused on training fine motor skills, such as improving their grasps and learning new grasps. Thus, instead of using a camera-based input they might benefit more from using tangibles.

\section{Test Setup and Methodology}

A case study was done in collaboration with the Sint Maartenskliniek (Nijmegen, the Netherlands), a hospital that specialises in posture and movement. This hospital offers specialised therapy for children between two and a half and eight years old with mild forms of unilateral CP. The therapy focuses on improving their affected hand and arm coordination and functioning. It consists of playful exercises such as playing board games and singing songs. During these exercises, they repetitively move their hands and arms. An example of such a therapy is the one called Pirate Group, in which the context of pirates is used to engage the children in the therapy. This is a type of modified Constraint Induced Movement 
Therapy combined with Bimanual Training intervention (mCIMT-BiT); children are forced to use their affected arm in the first few weeks, but later on they are allowed to use both hands in order to train their coordination. For further details of the therapy, please refer to [1].

As understanding both the therapy and the target group requires detailed knowledge, we chose to use a participatory design method. We implemented this throughout the design process: in gathering requirements, during the generation and development of concepts and finally in the evaluations. This was done in an iterative fashion in two main design rounds. The first round contained five subiterations and we made small changes to the games in each sub-iteration. In this first round of development we investigated several game dynamics using three games, whereas the second round focused more on therapists' and children's experiences.

We included spoken instructions in all games. The games made use of logging functionality to automatically record the time needed to finish a game. After the children finished the games, we asked them some questions, e.g. "Which game would you like to play again?". We also asked therapists for their feedback, focusing on movements and applicability. Below we will further explain the methodology used, and describe both the participants and the setting. In the next section, the games created will be described.

\subsection{First Round}

In the first sub-iteration we started with listening closely to the therapists to get to know the therapy, the target group and to assemble the requirements for the games. To this end, we held several interviews and informal discussions with four different therapists. In addition, an observational study was done by actively participating in two therapy sessions of one single group of five children with CP. To aid understanding of the therapists and to make communication easier, we did a literature review on CP. During the observational sessions, we witnessed the interests of the children closely and recorded these by taking notes. These interests were then used as basis for the first three games. These games were designed using a mixture and adaptation of design techniques involving physically acting out movements, combined with the use of objects lying around 348 : we were able to generate three games by repetitively acting out the tobe-trained movements. By using body movements, we tried to reduce the gap between creating games and triggering the proper movements. For further details about this methodology, please refer to 6 . We programmed the games using the SDK for TagTiles 10. We finished the first sub-iteration by showing a video prototype to one of the therapists.

In the second sub-iteration we tested the games informally on ten non-CP participants, mainly to find bugs and to improve game play. These tests were done in a home setting.

The third sub-iteration consisted of an evaluation with end users to test the suitability of the games and the triggered movements. We did the tests of the third sub-iteration in a classroom-like environment, containing one or two 
therapists, one researcher and one or two participants. Six children participated: three boys and three girls, all between four and six years old.

The fourth sub-iteration consisted of an in-subject test with four children. We used this evaluation to gain more insight into three game variables: sound, challenge and inter-personal competition, with a focus on the latter two. Because of the target group's reduced self-image it is important to choose the proper level of challenge which could help to increase their feeling of competence. Competition and cooperation are also relevant to that feeling. However, using one TagTiles system with two children at a time is impractical. In that case, the interactive space is too small to effectively train movements and using a turn-taking approach will reduce effectiveness of therapy as intensity is an important factor. Competition is also stimulated by adding a scoring system [16. The children played two variants of two games to analyse the effect of challenge and competition; these versions and the results will be described in Section 5. They played the "A-versions" in the first session and the "B-versions" in the second session or vice versa. The participants of this fourth sub-iteration did their tests in a small room with one participant, one therapist per child and a researcher present. The children were one 8 year old boy and three girls, two of 9 and one of 8 .

In the fifth sub-iteration, another group of children tested the games. The participants of the fifth sub-iteration used the same setting. The five children were an 8 year old girl, a 5 year old boy and girl, a 21/2 and a 3 year old girl. They played only one version of the game.

\subsection{Second Round}

We started the second round with a more thorough cooperative design session, to better include the therapists also during the creation process. During this session, two therapists developed several ideas using two design techniques in less than an hour. This design session was done with a physiotherapist and an occupational therapist in one of their offices.

The first design technique used is interaction relabelling [8]. In this technique, interactions of an object are translated to a new object, or in our case translated to a tangible game. Based on a first test session, with three other participants (non-therapists), it was decided to make use of objects with a mechanical character to efficiently trigger creativity. We used a stapler, a multi-head screwdriver, a Rubik's Cube and a small plastic box with a magnetic fastening. Participants were told to take the first prop in their hands and explain how they would use this. Based on this explanation they had to come up with ways to alter this into a game, training the wanted movements.

The second technique was a shortened version of Systematic Inventive Thinking (SIT). SIT is a way to alter a product, or to overcome a problem, by using effective often reoccurring thinking patterns [1]. SIT consists of the following steps:

- identifying the components of the idea/product,

- applying one of the thinking patterns, namely division, multiplication, subtraction, (task) unification or (change of) attribute dependency, 


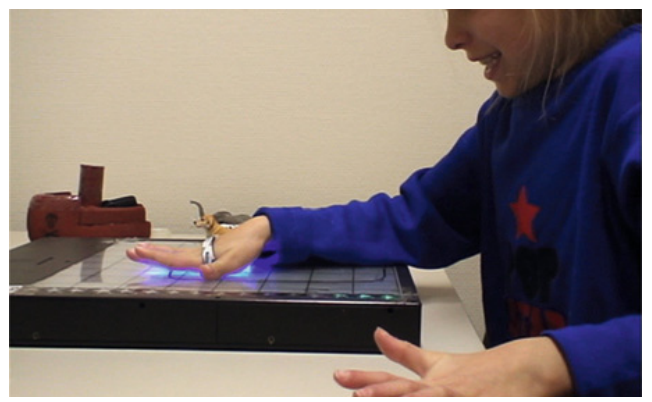

Fig. 1. A nine year old girl laughing while playing the hitting game

- visualising this solution,

- foreseeing whether it will fulfil the (new) needs.

For both techniques, a small explanatory presentation of 5 minutes was given to the therapists just before using the technique. Interaction relabelling was used for 20 minutes to create ideas followed by 25 minutes of SIT to further develop some of those ideas. Based on the outcome of this session, one game was created, incorporating the input of the therapists.

This game was tested by observing children playing the game. Three children participated: two 8 year old boys with reasonable hand and arm functionality and one 8 year old girl with more limited capabilities. The classroom setting of the second sub-iteration in the first round was used again. Children had to play the game for 15 to 30 minutes until a good image of the interaction was formed by direct observations. Following the gameplay, two therapists were interviewed. This included one therapist that observed all the three participants playing. The other therapist, who also participated in the design session, observed only two of the participants.

\section{Games}

Three games were created in the first round: a hitting game, an animal game and a boat shooting game. In the second round, a quiz game was developed. The games, the movements they trigger and the origin of the used themes will be described in this section.

\subsection{Hitting Game}

The hitting game is a simple game based on triggering rotation of the hand backwards. Children have to wear two hook and loop fastener strips for sensing purposes, one fastened around the wrist and one around the palm of the hand; see Fig. 11 One of the squares is lit yellow for about two seconds and then 'jumps' to the next square. While keeping their arm on the board they have to raise their palm and then hit the lighted square in time. On hitting the square, one of ten 


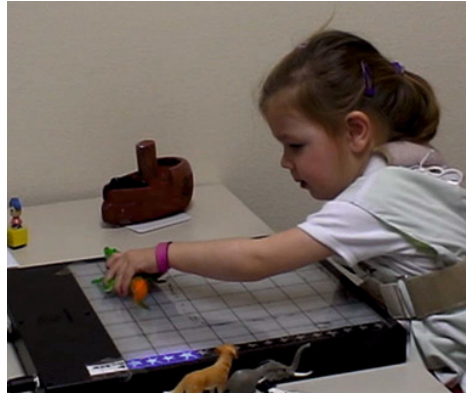

Fig. 2. A young girl playing the animal game

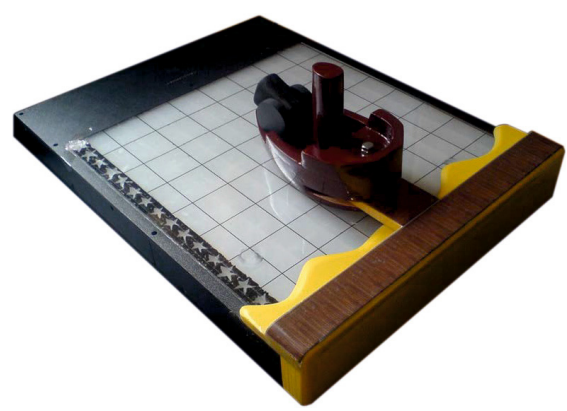

Fig. 3. The final version of the tangibles used for the boat shooting game

'funny' sounds is played and a point is awarded. If the wrist is raised while hitting, this is indicated by playing a spoken text. Once 16 points are achieved the game is won and the border of the board flashes green for several seconds.

\subsection{Animal Game}

During the observations, children were seen to enjoy making animal sounds and listening to how others made animal sounds. This was used as the inspiration for the animal game; see Fig. 2] In this game, the name of a colour is played, e.g. "Brown", then the child has to pick a miniature animal and place it on the board at the square lit in this colour. On placing the proper animal, either an indication that the child performed well is played, for example "Well done, a dog" or an animal sound is played. All the animals have to be placed and removed from the board to finish a round; the game consists of two rounds. If a child takes too long to place the animal, e.g. when losing concentration, a reminder is given "Which animal is brown?". This is followed by indicating the right animal after some seconds with "The doggy is brown". When a child places the wrong animal, this is also stated: "That is not the right animal". The game requires extension of the elbow and trains several grasps. We tried to pick the right variety of shapes and weight to trigger several grasps and to reduce the possibility of compensating movements.

\subsection{Boat Shooting Game}

Fitting the topic of the Pirate group, this game involves rotating a boat to shoot incoming targets. In the game created, targets slowly move down and a pirate boat has to be turned into the direction of the target; see Fig. 3. Aiming in the right direction results in destroying the target and one of two explosion sounds being played. The rotation of the boat requires rotating the hand backwards. To make this boat game playable for children with very limited ability to move their hand backwards, the board was rotated between 0 and 45 degrees, depending on the maximum angle the child showed to be able to manage. 


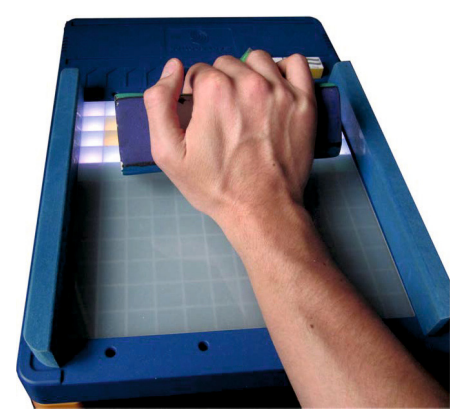

Fig. 4. Hearing the answers in the "Ik hou van Holland" game: tumbling a block, targets a passive form of rotating the hand backwards

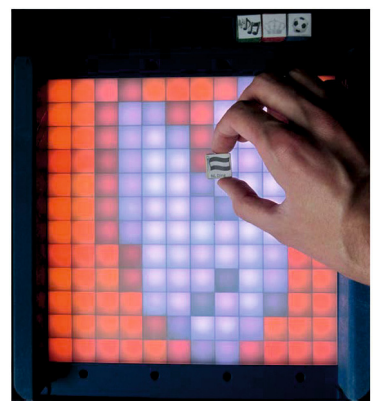

Fig. 5. The start of the "Ik hou van Holland" game. Placement of a wooden cube to select a category which triggers the shown "pincher grasp"

\subsection{Quiz Game}

This game is based on answering multiple choice questions. It is implemented on a newer version of TagTiles; see Fig. [. To answer the questions, several actions have to be performed, that trigger several movements. The game consists of: picking a category, getting a random question, hearing the options, choosing an answer, loading this answer and then hearing if it is correct or not. With the selection of actions and objects we tried to minimise compensating movements. The group from which three participants were selected in the evaluation of this game participated in (mCIMT-BiT) therapy with "Ik hou van Holland" as a metaphor. This is a Dutch TV-show in which Dutch TV personalities answer questions about Dutch habits, songs and personalities.

The game created starts with the intro tune and shows a tulip symbol. A category of questions has to be selected using a small wooden cube; see Fig. [0. This action requires a correct grasp of a small object triggering the pincher grasp; see Fig. [5]

An instruction is played to tip a question tag onto the board from the question box. The tipping movement triggers rotating the arm inwards and triggers outward rotation when placing it on the table again.

Four multiple choice answers can be heard by rotating a block over the board. This triggers rotating the hand backwards; see Fig. 4. An instruction is given to select the correct answer by placing an answer disc onto the board. This action also triggers the pincher grasp.

The user is instructed to load the answer. The answer has to be put into a tube. The user is then instructed to throw the 'answer' on the board. If the answer is wrong, the game proceeds again from hearing the question. If a question has been answered incorrectly for the second time or the correct answer is given, then the game starts again with selecting the category.

When all questions from a category are answered and this category is chosen, the user is instructed to select another category. If this happens, then the score 
is shown on the board. At the start of the game, the therapist or user can select the number of questions that have to be answered ranging from 5-12. When all answers from one category and the total number of questions are answered, the user hears that he or she did very well to answer all these questions correctly. This is followed by 20 seconds of the Queen song "We are the champions".

\section{$5 \quad$ Evaluation and Results}

Several evaluations have been done throughout the research. The most important of these will be described in this section. We have little to say about the use of a video prototype of a preliminary version in the first iteration. It was useful as it did not require fitting in a co-located meeting in a tight schedule. Also, it gave an early indication of whether the games would work. As for the second sub-iteration: testing with non-CP participants we found bugs and made improvements without 'bothering' children or therapists. Nine changes were incorporated as a result of these tests.

\subsection{Game Design Variables}

We researched three game design variables: sound, competition and challenge. We used an in-subject test in the fourth sub-iteration for the latter two.

Sound. In the third sub-iteration, we let some of the children play the hitting game without sound. We used direct observations of a researcher and therapists. Not having sound reduced the clarity of feedback and the entertainment drastically, thus in the forth and fifth sub-iterations we only used the version with sound. Analysis of videos taken in the fourth sub-iteration showed that three of the four children grinned upon hearing the first or second sound in the hitting game; see Fig. प Including the video analysis of the fifth sub-iteration of the first round as well, six out of nine children grinned or smiled as soon as they heard the first or second sound.

Competition. We compared two versions of the animal game to evaluate influence of competition. In the "A-version" of the animal game we introduced a competitive objective in two ways: (1) we instructed the children to finish as quickly as possible and to make as few mistakes as possible, (2) we introduced feedback on high scores after finishing the first and the second round of a game. In the "B-version" we excluded both forms of competition. The participants of the fourth sub-iteration did not give clear responses after hearing their results. The first participant sighed deeply and moved with her chair from left to right. The third participant stated he found the animal game too simplistic. We had expected that the encouragement would result in significantly faster finishing of the second round of the animal game. However, such an effect could not be observed. 
Challenge. We compared two versions of the boat game. The "A-version" with a higher level of challenge by targets coming down faster after each hit. The game also goes into a game over state when a target reaches the bottom of the board. The "B-version" did not have such an increase of speed or game over state. Two of the girls of the fourth sub-iteration smiled after losing the "A-version". In the interview, they both stated they disliked the game (both versions), they disliked that it was so difficult to aim in the required direction. One girl laughed after winning the pirate boat game after having lost the altered version during her previous session. One boy had some difficulty playing the game mainly due to the version of the tangible boat used. He could not finish it on his own and showed no clear reaction after losing the game. The challenge in the boat game was high, both handling the tangible object and aiming it in time, so all participants of the fourth sub-iteration lost the altered game. No clear indications of difference in perceived entertainment value was seen between the two versions of the game.

\subsection{Use of SIT and Interaction Relabelling}

Using interaction relabelling resulted in generating eight ideas. From these ideas, four were chosen in conjunction with the therapists to further develop, each using one or two randomly selected thinking patterns. Applying and understanding the thinking patterns was found to be more difficult than using interaction relabelling. The SIT-session nonetheless resulted in ten simple alterations. We implemented these proposed game elements and movement-object combinations in the quiz game.

\subsection{Age Dependency}

Based on the fourth sub-iteration (in-subject tests) and the fifth sub-iteration combined, taking everyone's first attempt and using an independent samples one-tailed t-test we could see a clear age difference in completion time for the animal game $(p<0.001)$ and the hitting game $(p<0.001)$ between $2-3$ year olds $(n=2)$ and $5-9$ year olds $(n=7)$. For the hitting game, there was also a significant difference between 5 and 8-9 year olds and between 2-5 and 8-9 year olds(both $p<0.05)$ ).

The second youngest child needed help in hitting the first target in the hitting game. In the animal game, someone had to put the correct animal in her hand and she needed additional instructions from the therapists. The pirate game was played together with the therapist but even in this way she could not understand it enough to play the game. Similar problems occurred with the youngest child. She often had to be directed towards where the target was in order to keep her attention on the game. During the animal game, the animals either had to be pointed out or handed to her. Several times she had a difficult time placing the animal upright on its pedestal.

The second youngest child showed clear use of the unwanted extreme (palmar) flexing of the wrist when placing some of the animals. However, the youngest did perform a proper grasp and placement by herself, see Fig. 2 . 


\subsection{Liking, Triggered Movements and Compensating Movements}

Responses from the children were quite positive. In the first round, one of the children of the third sub-iteration even spontaneously mentioned she would like to have the game at home. In the fourth and fifth sub-iterations, the hitting game and animal game were the most preferred (both 4 times). The youngest participant preferred the boat game. After being encourage by a therapist, she acknowledged this was because she liked the attractive tangible object. The therapists especially liked the animal game.

The children in the second round were positive about the quiz game. One of the boys, even without encouragement, kept on playing. Both boys replied that they would like to play the game again. The girl stated she did like the game more than other exercises she had done in therapy thus far. One of the participants found the questions a little too hard, though another did not mind that he did not know all the answers.

According to the three therapists present during tests in the first round, all targeted movements with the exception of rotating the arm outward were triggered. Turning the arm outward was triggered later on in the quiz game, for instance by putting the answer tube next to the board. In the discussion, both therapists concluded that especially the children with better arm-functions made the proper movements. Some forms of compensation were seen. For example, in the first sub-iteration a child moved the boat to the targets instead of turning it. In the boat game, extreme (palmar) flexing of the wrist was also seen. We fixed the boat to an axis in the latest version of the boat game to prevent these things from happening; see Fig. 3. With loosening or tightening the axis we could also adjust to the strength of the child. The animal game was mainly used as intended with a minimum of compensation, except for the second youngest child. In the quiz game, another girl -being slightly less capable- made more compensating movements than the two boys. For instance, she had to be reminded several times to perform the actions with her affected arm. Also, instead of turning the answer block she tumbled it by gently hitting it.

One of the therapists liked the feature that multiple movements were triggered in one game, especially if the game could be adapted to the player's personal functioning, for example by skipping parts of the game. The newer version of TagTiles was also liked, as it did not need an external laptop. This made it more mobile and reduced preparation time.

\section{Discussion}

Based on our research findings, we can draw several conclusions and make proposals for future research. Our findings can be very useful for future research in the field of tangible games, serious gaming for physical therapy and in general for designing interactive systems for people with specific needs. 


\subsection{Methodology}

We found that design techniques with acting out of movements were very applicable for designing physical therapy games. It certainly seemed to reduce the gap between training movements and developing a game. The relabelling in the participatory design session of the second round seemed useful and also very applicable. However, SIT seemed to need some more time to comprehend and use efficiently. Children, especially those with special needs such as children with $\mathrm{CP}$, form a diverse and hard to reach target group. This makes it difficult to do proper research. We found that one should be very careful in generalising for instance the capabilities of the children.

\subsection{Utilisation of Traditional Game Variables for CP Therapy}

Sound brings additional fun to games. Increasing challenge to the extend that one sometimes loses a game, might add extra motivation and feelings of fulfilment and capability when in the next attempt one does win. For instance, one girl showed a very enthusiastic response winning after losing first. Losing a game did not lead to extreme reactions like crying. Our addition of an inter/intra-personal competition feature by using high scores did not lead to noticeable effects in our tests.

\subsection{Using Tangibles for Occupational and Physical Therapy}

We found significant differences in the target group, for instance between age groups, hence we think it is essential to make games adaptable. We did some tests in which therapists could change settings in a GUI, such as timings and which animals to use, but the results were inconclusive. Based on interviews we came to the conclusion that if one instead uses a purely adaptive system, it might fail on some occasions. A system will have a hard time recognising the difference between lost attention (so a possible lack of challenge) and low capabilities (so too challenging). One solution is an adaptable adaptive system: one that can automatically change the game within the limits of a specific user.

We succeeded in creating an entertaining set of games that can bring additional motivation and fun in the context of occupational and/or physical therapy for children with CP. The games succeeded in training the correct movements related to goal-directed activities. Especially the fourth game, being a physical quiz created for the latest version of TagTiles, seems very promising. The children kept on playing the game, mainly making the proper movements without additional encouragement by or instructions from the therapists or researcher. However, some compensation still occurred, such as the tendency to grab objects with the non-affected hand during the quiz game and (palmar) flexing of the wrist too far in both the animal game and the boat game.

This kind of compensating movements remains a problem especially if one prefers partially unsupervised therapy. According to one of the therapists, a more mechanical device strictly guiding the movements might be more suitable 
for children with more limited physical capabilities, as compensating movements can be inhibited in such a device to a larger extent. On the downside such approaches usually require additional setup time and have higher costs. Using other less or non-tangible interaction techniques, such as the Kinect or Wii, might be more suitable for detecting and training some movements [5], for instance for the turning of the underarm outwards. Even if a child cannot grasp an object, it can still benefit from training other kinds of movements but might not manage with tangibles alone. However, using tangibles can trigger training of grasps quite well -as demonstrated in our research.

\subsection{Future Work}

Ongoing research by the consortium called Wikitherapist will further investigate the opportunities and limitations of using the TagTiles platform in combination with end-user development for occupational and physical therapy purposes. More quantitative measures about improvement in motor function using tangible games in therapy could help to encourage more widespread use. An interesting observation was the enjoyment in destroying virtual things. This was both stated in interviews and seen in observations. Although much research has been done on the effects of violence in games, we only found a limited amount of research investigating the link between fun and destroying virtual things. During several iterations, we found and solved both major and minor problems. However, we succeeded in triggering the correct movements and increasing motivation. It is considered worthwhile to further improve the quiz game with adaptability, adaptivity and incorporating additional game development principles, so as to make it an even more suitable and entertaining activity to use in occupational and physical therapy.

Acknowledgments. This publication was supported by the Dutch national program COMMIT. Intellident sponsored us with several RFID tags. Many thanks go out to: Julia Garde and Ronald Poppe for the pleasant discussions and their useful input, to the therapists of the Sint Maartenskliniek and to Serious Toys for providing information, help and the TagTiles used for this research.

\section{References}

1. Aarts, P.B., Jongerius, P.H., Geerdink, Y.A., van Limbeek, J., Geurts, A.C.: Effectiveness of modified constraint-induced movement therapy in children with unilateral spastic cerebral palsy: a randomized controlled trial. Neurorehabilitation and Neural Repair 24(6), 509-518 (2010)

2. Annema, J.H., Verstraete, M., Vanden Abeele, V., Desmet, S., Geerts, D.: Videogames in therapy: a therapist's perspective. In: Proceedings of the 3rd International Conference on Fun and Games, pp. 94-98. ACM, New York (2010)

3. Burns, C., Dishman, E., Verplank, W., Lassiter, B.: Actors, hairdos \& videotapeinformance design. In: Conference Companion on Human Factors in Computing Systems, CHI 1994, pp. 119-120. ACM, New York (1994) 
4. Buur, J., Jensen, M.V., Djajadiningrat, T.: Hands-only scenarios and video action walls: novel methods for tangible user interaction design. In: Proceedings of the 5th Conference on Designing Interactive Systems: Processes, Practices, Methods, and Techniques, DIS 2004, pp. 185-192. ACM, New York (2004)

5. Chen, Y.P., Kang, L.J., Chuang, T.Y., Doong, J.L., Lee, S.J., Tsai, M.W., Jeng, S.F., Sung, W.H.: Use of virtual reality to improve upper-extremity control in children with cerebral palsy: a single-subject design. Physical Therapy 87(11), 14411457 (2007)

6. van Delden, R.W.: Design of therapeutic TagTile games for children with unilateral spastic cerebral paresis. Master's thesis, University of Twente, the Netherlands (September 2011)

7. Deutsch, J.E., Borbely, M., Filler, J., Huhn, K., Guarrera-Bowlby, P.: Use of a low-cost, commercially available gaming console (Wii) for rehabilitation of an adolescent with cerebral palsy. Physical Therapy 88(10), 1196-1207 (2008)

8. Djajadiningrat, J.P., Gaver, W.W., Fres, J.W.: Interaction relabelling and extreme characters: methods for exploring aesthetic interactions. In: Proceedings of the 3rd Conference on Designing Interactive Systems: Processes, Practices, Methods, and Techniques, DIS 2000, pp. 66-71. ACM, New York (2000)

9. Griffiths, M.: The therapeutic use of videogames in childhood and adolescence. Clinical Child Psychology and Psychiatry 8, 547-554 (2003)

10. van Herk, R., Verhaegh, J., Fontijn, W.F.: Espranto sdk: an adaptive programming environment for tangible applications. In: Proceedings of the 27th International Conference on Human Factors in Computing Systems, CHI 2009, pp. 849-858. ACM, New York (2009)

11. Horowitz, R., Maimon, O.: Creative design methodology and the sit method. In: Proceedings of DETC 1997: 1997 ASME Design Engineering Technical Conference, pp. 14-17 (1997)

12. Kannetis, T., Potamianos, A.: Towards adapting fantasy, curiosity and challenge in multimodal dialogue systems for preschoolers. In: Proceedings of the 2009 International Conference on Multimodal Interfaces, ICMI-MLMI 2009, pp. 39-46. ACM, New York (2009)

13. Kierkegaard, P., Markopoulos, P.: From Top to Bottom: End User Development, Motivation, Creativity and Organisational Support. In: Costabile, M., Dittrich, Y., Fischer, G., Piccinno, A. (eds.) IS-EUD 2011. LNCS, vol. 6654, pp. 307-312. Springer, Heidelberg (2011)

14. Korhonen, H., Montola, M., Arrasvuori, J.: Understanding playful user experience through digital games. In: Guenand, A. (ed.) Proceedings of the 4th International Conference on Designing Pleasurable Products and Interfaces, DPPI 2009, pp. 274-285 (2009)

15. Li, Y., Fontijn, W., Markopoulos, P.: A Tangible Tabletop Game Supporting Therapy of Children with Cerebral Palsy. In: Markopoulos, P., de Ruyter, B., IJsselsteijn, W.A., Rowland, D. (eds.) Fun and Games 2008. LNCS, vol. 5294, pp. 182-193. Springer, Heidelberg (2008)

16. Malone, T.W., Lepper, M.R.: Making learning fun: A taxonomy of intrinsic motivations for learning. In: Snow, R.E., Farr, M.J. (eds.) Aptitude Learning and Instruction: III Conative and Affective Process Analyses, vol. 3, pp. 223-253. Lawrence Erlbaum Associates, Hilsdale (1987)

17. Moon, H.K., Baek, Y.K.: Exploring variables affecting player's intrinsic motivation in educational games. In: Proceedings of the 17th International Conference on Computers in Education, pp. 718-722 (2009) 